\title{
Internet-Delivered Cognitive Behavioural Therapy in a Canadian Community Hospital: A Novel Approach to an Evidence-Based Intervention
}

\author{
David Gratzer \\ Centre for Addiction and Mental Health \\ Faiza Khalid-Khan \\ Ontario Shores Centre for Mental Health Sciences \\ Shawnna Balasingham \\ Centre for Addiction and Mental Health \\ Nadia Yuen, and Janany Jayanthikumar \\ Scarborough and Rouge Hospital
}

\begin{abstract}
To increase access to evidence-based care, people with depression and anxiety in a Canadian community hospital were offered internet-delivered CBT (iCBT) or in-person CBT. The iCBT outpatients had a lower attrition rate with similar symptom reduction. Our results suggest that other clinics and hospitals could use iCBT to facilitate care.
\end{abstract}

Keywords: Cognitive Behavioural Therapy (CBT), Internet-delivered CBT (iCBT), psychological intervention, depression, anxiety, access

David Gratzer, Scarborough and Rouge Hospital, Toronto, Ontario, and the Department of Psychiatry, University of Toronto, Toronto, Ontario; Faiza Khalid-Khan, Scarborough and Rouge Hospital, Toronto, Ontario; Shawnna Balasingham, Scarborough and Rouge Hospital, Toronto, Ontario; Nadia Yuen, Scarborough and Rouge Hospital, Toronto, Ontario; Janany Jayanthikumar, Scarborough and Rouge Hospital, Toronto, Ontario.

David Gratzer now has additional affiliation at the Centre for Addiction and Mental Health, Toronto, Ontario; Faiza KhalidKhan is now at Ontario Shores Centre for Mental Health Sciences, Whitby, Ontario; Shawnna Balasingham is now at the Centre for Addiction and Mental Health, Toronto, Ontario

No financial support to disclose.

Correspondence concerning this article should be addressed to Dr. David Gratzer, Centre for Addiction and Mental Health, 250 College St., Toronto ON M5T 2S1.Email: David.Gratzer@camh.ca 


\section{RÉSUMÉ}

Afin d'offrir des soins appropriés à un plus grand nombre de personnes souffrant de dépression et d'anxiété, un hôpital communautaire de la région de Toronto a proposé à ces personnes la possibilité de suivre une thérapie cognitivo-comportementale en personne ou par Internet. Dans cet article, nous montrons que, chez les patients ayant suivi une thérapie par Internet, la réduction des symptômes a été la même que chez les patients ayant choisi une thérapie conventionnelle, mais que le taux d'abandon a été inférieur. Nos résultats suggèrent donc que, dans d'autres cliniques ou hôpitaux, l'offre d'une thérapie par Internet pourrait être une façon d'accroître l'accès aux soins.

Mots clés : thérapie cognitivo-comportementale, thérapie cognitivo-comportementale par Internet, intervention psychologique, dépression, anxiété, accès.

The Scarborough Hospital (TSH), now part of the Scarborough and Rouge Hospital, is a large community hospital serving Toronto's diverse east end; the mental health department receives roughly 500 outpatient referrals per month. The authors have been actively involved in the development and implementation of an internet-delivered CBT program - the first such program in a Canadian community hospital, to our knowledge. Though there is an international body of literature for iCBT, little work has been done in a general outpatient setting to date; additionally, program implementation in Canada has a unique challenge in that it is not the traditional way of accessing care, and is therefore different than client expectations. In this paper, we outline the program and the lessons learned, and then consider the possibilities for expanding this program.

\section{THE NEED FOR ICBT}

The effectiveness of psychological interventions is well established for mental disorders. Cognitive Behavioural Therapy (CBT), for example, is as effective for mild and moderate major depressive disorder as are medications; according to Canadian guidelines, combined psychopharmacology and CBT for depression is superior to either modality alone, suggesting a synergistic effect (Parekh et al., 2016). But CBT requires significant resources, partly explaining its limited availability. In a survey of British Columbians, Puyat, Kazanjian, Goldner, and Wong found that only $13 \%$ of people with depression received any form of therapy or counselling (Puyat et al., 2016).

In 2013, the TSH Mental Health Outpatient Program was overhauled to increase access to evidencebased interventions. CBT was chosen as the main psychological intervention, and staff were trained in CBT (including CBT coursework though McMaster University and ongoing supervision). CBT was offered both in individual and group settings. To enhance access, evening hours were offered. Despite the offer of evidencebased therapy provided at no cost at the point of use, and extended clinic hours, many people didn't engage in any CBT, while others did not complete the course.

The current authors wondered if technology could be used to help with such access and attrition challenges. Internet-delivered CBT (iCBT) is intensely researched, with various studies showing that therapist-guided iCBT can equal traditional (in-person) CBT outcomes (Gratzer \& Khalid-Khan, 2016), with particularly robust 
evidence for depression (Thase et al., 2017); additionally, several countries have experimented with iCBT as part of their publicly funded services (Gratzer \& Goldbloom, 2016).

In collaboration with Queen's University (where a small iCBT program for adolescents was piloted), modules were developed that closely mirrored in-person CBT. The iCBT model consisted of a therapistguided internet version, which outpatients completed on their own schedule and from a location of their choice. This project was approved by the TSH Research and Ethics Board.

\section{Implementation: iCBT 1.0, January 2014-December 2014}

In iCBT 1.0, outpatients were provided eight modules containing the main principles of CBT, including the importance of noticing the connections between thoughts, feelings, and behaviours, scheduling mastery and pleasurable activities, utilizing breathing and relaxation exercises, reviewing thought logs, and restructuring inaccurate thoughts. All therapists (with backgrounds in psychology and social work) in the Outpatient Program have been trained in CBT, however, those selected to provide iCBT were given four hours of additional training by a Queen's University physician, where they were taught to provide CBT through electronic modules and worksheets. Outpatients were assigned a therapist who communicated with them through email. Prior to starting the program, all participants signed a confidentiality form agreeing that the modules, as well as the therapist and client responses, would be sent through email (and this was approved by the TSH Risk Management Officer). Participants were sent modules on a weekly basis by email, then expected to complete the attached worksheets within the week and email them back. The therapist would review the completed worksheets and provide feedback that would help the outpatient restructure their thoughts and increase their participation in helpful activities.

A review at six months showed disappointing results. The attrition rate was $90 \%$; many outpatients reported that they did not find the program helpful.

To understand the high attrition rate, patients were contacted (both those who completed and those who didn't complete the program). They were asked questions including, "Why did you drop out?" A common theme emerged: people found that the program was too rigid. Some felt that the deadlines for homework completion were too anxiety provoking. The pace was overwhelming for some, so they did what made them feel better- they avoided the program. Others who were more skilled at such homework found the pace too slow. iCBT 1.0 was designed without user input, and the result was a program that was not user-friendly.

This feedback was incorporated into a redesigned program that was launched two months later.

\section{Implementation: iCBT 2.0, February 2015-April 2017}

Several steps were taken to improve iCBT. Among them: the modules were streamlined to a total of six modules (down from eight); more examples and visuals were incorporated to highlight the material; fewer rigid deadlines were imposed, and an effort was made to make the program more flexible. To the latter point: outpatients worked with their internet-based therapists to determine timeframes that best worked with their schedules and symptoms. For those whose symptoms were less distressing, one or two modules per week were provided, and for those who were struggling more with their symptoms, deadlines were waived. But 
no matter the pace, therapists checked in with outpatients at least weekly to offer support and answer questions. Phone sessions were provided as well, if needed, to better explain the concepts and to troubleshoot.

To determine the effectiveness of iCBT, outcome measures were collected using the short version of the Depression, Anxiety, Stress Scale (DASS 21) and the Quality of Life Enjoyment and Satisfaction Questionnaire (Q-LES-Q). Inclusion criteria included diagnoses of depressive and/or anxiety disorders, as determined by unstructured psychiatric interviews using the criteria of the Diagnostic and Statistical Manual of Mental Disorders, Fifth Edition (DSM-5; American Psychiatric Association, 2013). Exclusion criteria included psychotic symptoms and lack of internet access. All participants were 18 years of age or older. In-person CBT had the same inclusion and exclusion criteria.

\section{RESULTS}

We enrolled in iCBT 120 people who met the inclusion and exclusion criteria; of those, 80 completed the program. Participants had a variety of diagnoses including co-morbid anxiety and depressive disorders. Most participants were female (66.3\%), and the median age was 39.5 years ( $\mathrm{SD}=13.71$ years). The attrition rate was 33\%. Significant improvements in measures of depression, anxiety, and stress were noted. Feedback was very positive for most, if not all, participants. The in-person arm was demographically similar, and had 75 people enrolled.

Compared to participants in the in-person arm, the attrition rate in the iCBT arm was lower (33\% vs. $40 \%$ ), with what seemed to be larger reductions in symptom severity (depression, anxiety and stress), although these differences were not statistically significant using a paired t-test.

\section{Lessons Learned}

As interest in iCBT grows, with public and private payers considering this modality of care, several lessons that were learned are important to note.

First, a simple internet-delivered model didn't work. iCBT 1.0 had a high attrition rate. In contrast, iCBT 2.0 had a lower attrition rate than in-person CBT. User engagement is important, whether care is delivered online or in-person.

Second, many people found internet-based treatment preferable and more convenient than in-person treatment. For example, a 28-year-old woman with a history of anxiety stated that she wouldn't have been able to attend a weekly clinic in-person because of her anxiety about taking the bus. A 25-year-old woman with a major depressive disorder was able to participate in iCBT despite being away at university during the week. iCBT wasn't always a replacement for in-person treatment, but did offer another option for outpatients.

Third, the international literature suggests that iCBT is more efficient than in-person CBT (Gratzer \& Khalid-Khan, 2016), and while therapist time wasn't measured in this study, it did seem that therapists spent less time on the tasks associated with delivering the internet-delivered therapy than with delivering the more traditional method. 


\section{Strengths and Limitations}

In terms of strengths, iCBT was offered to a real-world population with minimal exclusion criteria, in a community hospital outpatient clinic which is similar to many mental healthcare settings in Canada and elsewhere; hence, our program and its findings may be transferrable. In terms of limitations, this study was not a randomized controlled trial. The early data did not include much demographic information for predictive analysis. Additionally, costs associated with the study were not reported.

\section{FUTURE DIRECTIONS}

The iCBT program offered convenient, evidence-based treatment. Participants showed improvement in attrition, symptom severity, and other measures, and the results of iCBT were comparable to or better than (though not statistically significantly) the results of those receiving in-person CBT. Clinician time efficiency may have been better for iCBT than for in-person CBT.

This project may be difficult to replicate given the small sample size and the levels of organizational commitment and staff enthusiasm required. This pilot program, however, points a way forward; a larger-scale and better-controlled study of iCBT in such Canadian and other settings is needed.

\section{REFERENCES}

American Psychiatric Association. (2013). Diagnostic and statistical manual of mental disorders (5th ed.). Arlington, VA: American Psychiatric Publishing.

Gratzer, D., \& Khalid-Khan, F. (2016). Internet-delivered cognitive behavioural therapy in the treatment of psychiatric illness. Canadian Medical Association Journal, 188(4), 263-272.

Gratzer, D., \& Goldbloom, D. (2016). Making evidence-based psychotherapy more accessible in Canada. Canadian Journal of Psychiatry, 61(10), 618-623.

Puyat, J., Kazanjian, A., Goldner, E., \& Wong, H. (2016). How often do individuals with major depression receive minimally adequate treatment? A population-based, data linkage study. Canadian Journal of Psychiatry, 61(7), 394-404.

Parekh, S., Quilty, L., Ravitz, R., Rosenbluth, M., Pavlova, B., Grigoriadis, S.,...Uher, R. (2016). Canadian network for mood and anxiety treatments (CANMAT). Clinical guidelines for the management of major depressive disorder in adults. Section 2. Psychological treatments. Canadian Journal of Psychiatry, 61(9), 524-539.

Thase, M., Wright, J., Eells, T., Barrett, M., Wisniewski, S., Balasubramani, G.,...Brown, G. K. (2017). Improving the efficiency of psychotherapy for depression: Computer-assisted versus standard CBT. American Journal of Psychiatry, 175(3), 242-250. 